\title{
Utilidad de la tromboaspiración manual de rutina durante la angioplastía primaria en la preservación del flujo microvascular
}

\author{
Martín Valdebenito, Dante Lindefjeld, Nicolás Veas, José Luis Winter, Pablo Antileo, Alberto \\ Fuenzalida, Sebastián Flores, Manuel Méndez, Osvaldo Pérez, Eduardo Guarda, Alejandro \\ Martínez. \\ Departamento de Enfermedades Cardiovasculares, Centro de Terapia Endovascular del \\ Hospital Clínico de la Pontificia Universidad Católica de Chile, Laboratorio de Hemodina- \\ mia Hospital Dr. Sótero del Río.
}

Antecedentes: La efectividad de la aspiración de trombos de rutina en una angioplastía primaria (AP) es controversial.

Objetivo: Evaluar la efectividad de la aspiración de rutinaria de trombos durante la AP en la preservación del flujo microvascular final, estratificada por horas de presentación.

Métodos: Realizamos un ensayo clínico prospectivo randomizado de 122 pacientes que se presentaron al Hospital Sótero del Río con infarto agudo al miocardio con upradesnivel del ST (IAM c/SDST) a AP + aspiración o sólo AP, evaluando el flujo microvascular final.

Resultados: Se randomizaron 59 pacientes a AP + aspiración y 63 a sólo AP. En el grupo de aspiración hubo menor empleo de pre-dilatación $(46.4 \%$ vs $80.3 \%$; $\mathrm{p}=0.01)$ y menor necesidad de realizar técnicas comple- jas de bifurcación (3.6\% vs 15.2\%; p=0.03). No observamos diferencias en el flujo microvascular final medido por conteo de cuadros TIMI (cTFC) (25.6 \pm 11.8 vs 24.8 \pm 10.9 ; $\mathrm{p}=0.12$ ). No hubo diferencias en el flujo microvascular final, de acuerdo al tiempo de presentación: $<3$ horas de IAM c/SDST (cTFC $22.3 \pm-9.1$ vs $22.4 \pm-8$; $\mathrm{p}=$ $0.2) \mathrm{y}>3$ horas de IAM c/SDST (cTFC $27.7+/-13.4$ vs $25.9+/-11.6 ; \mathrm{p}=0.08)$.

Conclusion: En nuestra experiencia, no hay mejoría del flujo microvascular final en la tromboaspiración manual de rutina durante la AP como tampoco en el subgrupo de presentación precoz. La menor predilatación y el menor uso de técnicas complejas de bifurcación, podría traducir una angioplastía técnicamente más fácil al aspirar el trombo y permitir una mejor visualización del vasoculpable.

Correspondencia:

Dante Lindefjeld S

División de Enfermedades Cardiovasculares - Pontificia Universidad Católica de Chile.

Fono: +56987850098

dslindefjeld@gmail.com 


\section{Routine Thrombi Aspiration during Primary Anngioplasty does not improve micro vascular flow compared to conventional angioplasty in ST elevation Myocardial Infarction.}

Background. There is controversy regarding the effectiveness of thrombi aspiration during primary angioplasty for ST elevation myocardial

Aim: to evaluate the effect of routine aspiration of thrombus upon micro vascular flow, both overall and according to the time from initial pain to intervention.

Method: 122 patients admitted to a general hospital for acute ST elevation myocardial infarction were prospectively randomized to undergo coronary angioplasty (PTCA) with prior aspiration of thrombi or PTCA alone. The final micro vascular flow was compared between groups. A similar analysis compared patients presenting before or after 3 hours from de initial pain. Results: 59 patients underwent thrombi aspiration followed by PTCA (A+PTCA) and 63 PTCA alone (PTCA). Compared to the PTCA group, the A+PTCA group required less frequently balloon pre-dilation. ( $46.4 \%$ vs $80.3 \%$; $\mathrm{p}=0.01$ ) and a need for complex angioplasty of bifurcation lesions (3.6\% vs $15.2 \%$; $\mathrm{p}=0.03$ ). No difference was observed in final micro vascular flow assessed by cTFC between corresponding groups ( $25.6 \pm 11.8$ vs $24.8 \pm 10.9$; $\mathrm{p}=0.12$ ). Compared to Group A+PTCA, patients undergoing PTCA alone had no difference in final micro vascular flow, irrespective of time from initial pain to PTCA: cTFC $22.3 \pm-9.1$ vs $22.4 \pm-8$ in those treated $<3$ hours from initial pain and $27.7+/-13.4$ vs $25.9+/-11.6$, in those treated $>=3 \mathrm{hr}$ after initial pain, respectively.

Conclusion: Manual thrombi aspiration before PTCA in patients with ST elevation myocardial infarction did not improve micro vascular flow, regardless of the time from initial pain to primary PTCA. The lesser need for complex angioplasty and balloon pre-dilatation in patients undergoing thrombi aspiration may reflect a better visualization of affected arteries during PTCA.

Key Words: Myocardial Infarction; Angioplasty, Balloon, Coronary; Thrombosis 


\section{Introducción:}

La reperfusión miocárdica es el objetivo principal de una angioplastía primaria (AP) en un infarto agudo al miocardio con supradesnivel del ST (IAM c/SDST). ${ }^{1}$ Aparte de la restauración del flujo en la arteria coronaria epicárdica, últimamente se ha enfatizado la importancia de la microcirculación ${ }^{2,3}$. La falla de la reperfusión miocárdica se ha asociado con mayor tamaño de infarto, predisposición a arritmias ventriculares, insuficiencia cardíaca, shock cardiogénico, infarto al miocardio recurrente y muerte de causa cardiovascular ${ }^{4,5}$. Aunque la AP ha contribuido a una reducción importante en la mortalidad cardiovascular en las últimas tres décadas, la perfusión miocárdica normal no es restaurada en aproximadamente $1 / 3$ de estos pacientes. Varios mecanismos pueden contribuir a la falla de la reperfusión miocárdica, en particular la embolización distal de trombo y fragmentos de placa. En efecto, esta es una complicación posible durante una AP, resultando en una obstrucción de la microcirculación y fenómeno de no-reflow. La carga trombótica en el momento de una AP en pacientes con IAM c/SDST es asociado a peores resultados del procedimiento y clínicos, considerándose un predictor independiente de resultados adversos ${ }^{6-10}$. La embolización coronaria distal ocurre predominantemente en el momento de la inflación inicial del balón o stent, por lo que la reducción de la carga trombótica antes de la inflación del balón/stent puede disminuir la incidencia del fenómeno de no-reflow ${ }^{11}$. Los catéteres de aspiración manual son los más comúnmente usados, debido a que son fáciles y seguros de usar, incluso en la tercera edad ${ }^{12}$, y son relativamente menos costosos que los otros dispositivos como la trombectomía reolítica ${ }^{13}$. La evidencia actual sobre la utilidad de la aspiración de trombos durante una AP es controversial, tendiendo una recomendación IIa en las guías actuales. La mayor parte de la literatura, incluyendo meta-análisis y estudios clínicos randomizados, concluye que la trombectomía mejora los parámetros de reperfusión miocárdica ${ }^{14}$. Los estudio TAPAS y EXPIRA y algunos meta-análisis encontraron que la aspiración manual de trombos durante un IAM c/ SDST mejora la reperfusión miocárdica y los resultados del procedimiento, reduciendo el no-reflow y la embolización distal ${ }^{15-17}$. Sin embargo, el recientemente publicado estudio TASTE ${ }^{18}$ demostró ausencia de un beneficio clínico en mortalidad, re-infarto o trombosis del stent a 1 año de seguimiento. Actualmente, no existe evidencia suficiente para apoyar la tromboaspiración manual en el sub-grupo de pacientes que se presentan precozmente en un IAM c/SDST.
Nosotros realizamos un ensayo clínico randomizado para evaluar el efecto de la aspiración de trombos en la reperfusión miocárdica medido por flujo microvascular durante una AP. También se evaluó su utilidad de acuerdo al tiempo de presentación del IAM c/SDST.

\section{Métodos:}

Diseño del Estudio: Se realizó un estudio clínico prospectivo, randomizado, controlado, analizando los datos recolectados en el ingreso y durante el procedimiento. El diseño del estudio fue aprobado previamente por el comité de ética del Hospital Dr. Sótero del Río. El estudio fue diseñado y conducido por los autores, quienes escribieron el manuscrito y estuvieron de acuerdo en su publicación. No hubo financiamiento externo para la realización del estudio y ninguno de los autores tiene conflicto de intereses en relación al estudio.

Pacientes: Se enrolaron a los pacientes con IAM c/SDST tratados con angioplastía primaria en el Hospital Dr. Sótero del Río entre Agosto 2012 y Agosto 2014.

Los criterios de inclusión fueron: pacientes $>18$ años, que se presentaron con IAM c/ SDST sometidos a angioplastía primaria. Los criterios de exclusión fueron imposibilidad de revascularización, necesidad de cirugía de revascularización de urgencia y trombolisis reciente.

A los pacientes que cumplían los criterios de inclusión y exclusión, se les pidió el consentimiento informado, randomizándolos posteriormente en una razón 1:1 a aspiración o no de trombos con catéter Export. Dos operadores ciegos a la rama randomizada analizaron de forma independiente los resultados angiográficos de flujo microvascular (Flujo TIMI, Blush Miocárdico, conteos de cuadros TIMI (cTFC)). Si no existía consenso un tercer operador ciego analizaba los resultados.

Angioplastía Primaria: Se consideró una angioplastía primaria al procedimiento realizado en pacientes con angina $>30$ minutos y presencia de SDST $>1 \mathrm{~mm}$, en al menos dos derivaciones contiguas. Previo a la PTCA los pacientes con IAM c/SDST, recibieron en el servicio de urgencia una combinación de algunos de los siguientes agentes: aspirina, betabloqueadores, nitroglicerina, heparina o analgésicos. Durante la intervención se mantuvo un tiempo de coagulación (ACT) > 300 segundos y se administró $600 \mathrm{mg}$ de clopidogrel. El uso de inhibidores IIb/IIIa, nitroglicerina, adenosina, verapamilo, nitroprusiato, predilatación o postdilatación con balón quedó a discreción del operador. 


\section{Definiciones}

a) IAM c/SDST: Se definió como tal a la presencia de dolor torácico asociado a elevación del segmento ST, una onda $\mathrm{Q}$ patológica, un nuevo bloqueo de rama izquierda o una localización posterior del infarto con depresión del segmento ST en pared anterior y alza de biomarcadores (troponinas, CK Total y CK MB).

b) Flujo TIMI: Se clasificó el flujo epicárdico de una arteria coronaria en una coronariografía como 0: sin flujo anterógrado; 1: el medio de contraste pasa más allá de la obstrucción, pero de forma lenta y sin opacificar el lecho coronario distal; 2: el medio de contraste pasa más allá de la obstrucción y opacifica el lecho. coronario distal a la obstrucción; de forma lenta y con "clearence" lento; 3: el flujo anterógrado en el lecho distal ocurre de forma casi simultánea al lecho proximal y con rápido "clearence” del medio de contraste.

c) Para estimar la reperfusión se utilizó "Blush Miocárdico", método que valora la densidad de contraste en el territorio de miocardio dependiente de la arteria causante, como. 0: ausencia de densidad de contraste; 1 : mínima densidad de contraste; 2 : densidad de contraste moderada pero inferior a la obtenida durante la angiografía de una arteria contralateral o ipsilateral no relacionada con el infarto; 3: densidad de contraste normal, comparable a la obtenida durante la angiografía de una arteria contralateral o ipsilateral no relacionada con el infarto.

d) La estimación de carga trombótica en el vaso culpable del infarto se efectuó con la Escala de Carga de Trombos TIMI: Grado 0: sin evidencia angiográfica de trombo; Grado 1: Trombo posible, con densidad de contraste reducida, imagen de lesión con contorno irregular sugerente de trombo, pero no diagnóstico; Grado 2: trombo definitivo, con dimensión mayor similar o menor a la mitad del diámetro del vaso; Grado 3: trombo definitivo con dimensión mayor entre 0.5 a 2 veces el diámetro del vaso; Grado 4 trombo definitivo con dimensión mayor superior a 2 veces el diámetro del vaso y, Grado 5: oclusión total del vaso.

e) Conteo de Cuadros TIMI (cTFC): Número de cuadros cineangiogáficos que transcurren entre el ingreso del contraste al extremo proximal de la arteria y la llegada del mismo a las marcas anatómicas previamente determinadas para cada arteria. Un cTFC sobre 23 cuadros ha sido establecido como el punto de corte para definir mal resultado.

\section{Análisis Estadístico:}

Se usaron estadísticas descriptivas, tales como media, desviación estándar y frecuencia, que fueron calculadas para cada característica clínica y demográfica si era apropiada. Además, se utilizaron pruebas estadísticas de chi-cuadrado, test de Mann-Whitney, o t de Student según correspondiera. Un $\mathrm{p}<0.05$ se consideró como estadísticamente significativo.

Se analizaron por intención de tratar las características clínicas, angiográficas pre y post-procedimiento (Flujo TIMI, conteo de cuadros TIMI (cTFC), Blush miocárdico), comparando los resultados de la aspiración vs no aspiración de trombos. El mismo anásis se efectuó estratificado de acuerdo al tiempo de evolución del IAM c/SDST (menos o más de 3 horas, concordante al mejor punto de predicción de la curva ROC y de acuerdo a lo descrito en el estudio TAPAS). El análisis estadístico fue realizado usando el programa estadístico SPSS v16.0 (SPSS Inc., Chicago, IL).

\section{Resultados}

En el período seleccionado se reclutaron 122 pacientes, de los cuales se randomizaron 59 pacientes a tromboaspiración manual + angioplastía primaria y 63 a sólo angioplastía. Ambos grupos fueron comparables en sus características clínicas (Tabla 1) y angiográficas basales (Tabla 2) Destaca que en nuestros pacientes la mayoría fueron hombres con una edad promedio de 59 años, donde los principales factores de riesgo fueron la HTA y el tabaquismo. La duración promedio del dolor del IAM fue 5 horas y la mayor parte de los pacientes se presentaron en Killip

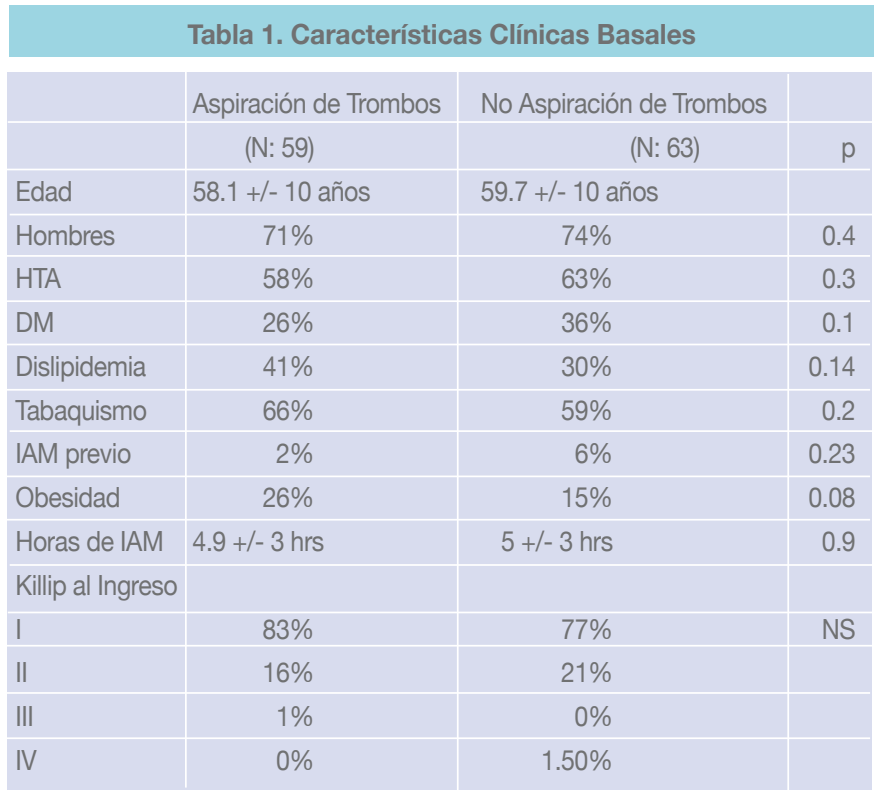

HTA: Hipertensión Arterial; DM: Diabetes Mellitus; IAM: Infarto Agudo al Miocardio; NS: no significativo 


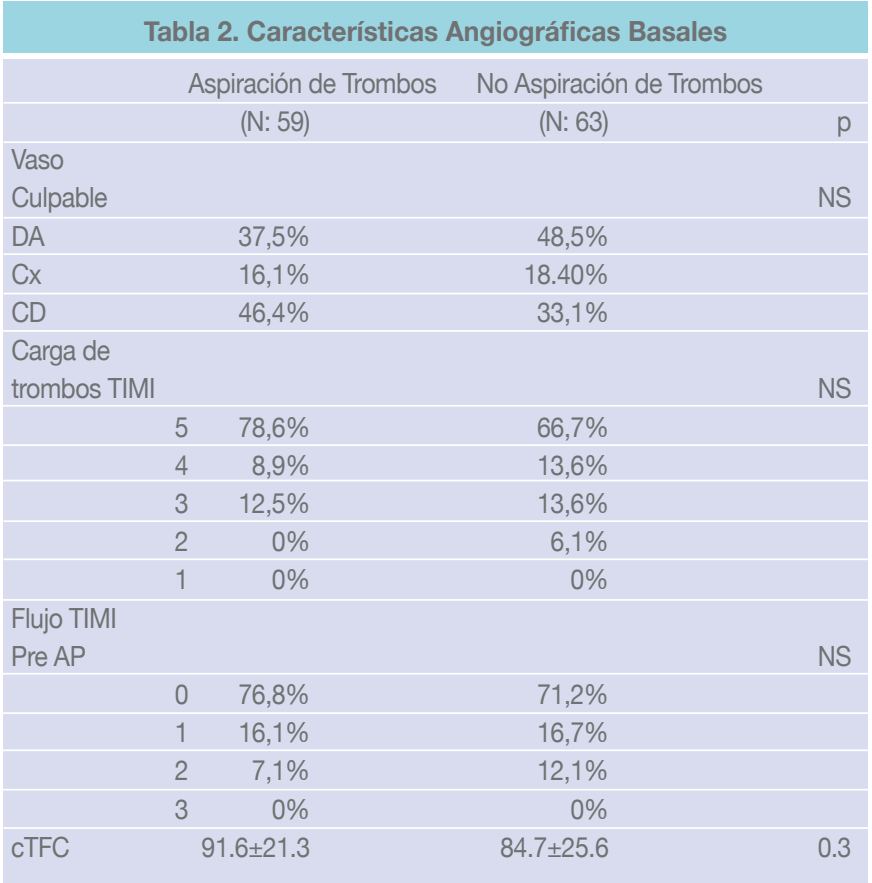

DA: Descendente anterior, Cx: Circunfleja, CD: Coronaria derecha, AP: Angioplastía Primaria, NS: no significativo.

I. La mayor parte tenía una alta carga trombótica y el flujo coronario inicial estaba deteriorado (TIMI, cTFC). Hubo crossover en ambos grupos, $8.9 \%$ en el grupo de aspiración y $12.1 \%$ en el grupo de no aspiración ( $\mathrm{p}=0.57$ ).

Las características periprocedimiento se presentan en la Tabla 3. Se puede observar que los pacientes sometidos a aspiración de trombos tuvieron menor necesidad de pre-dilatación de lesiones y menor necesidad de emplear técnicas complejas de bifurcación. No hubo diferencias en post-dilatación de lesiones ni en las complicaciones periprocedimiento, específicamente de la presencia de fenómeno de no-reflow.

\begin{tabular}{|l|r|r|r|}
\hline \multicolumn{3}{|c|}{ Tabla 3. Características Periprocedimiento } \\
\hline & $\begin{array}{l}\text { Aspiración } \\
\text { de Trombos }\end{array}$ & $\begin{array}{c}\text { No Aspiración } \\
\text { de Trombos }\end{array}$ \\
\hline & $(\mathrm{N}: 59)$ & $(\mathrm{N}: 63)$ & $\mathrm{P}$ \\
\hline Pre-dilatación & $46,4 \%$ & $80,3 \%$ & 0.002 \\
\hline Post-dilatación & $26,8 \%$ & $40,9 \%$ & 0.07 \\
\hline Técnicas de Bifurcación & $3,6 \%$ & $15,2 \%$ & 0.03 \\
\hline Fenómeno de no-reflow & $7,1 \%$ & $7,6 \%$ & 0,6 \\
\hline
\end{tabular}

$\mathrm{Al}$ analizar los resultados angiográficos finales de flujo microvascular, observamos que no hubo diferencias entre ambos grupos en cuanto a las variables cualitativas de flujo microvascular (flujo TIMI final, Blush miocárdico) ni cuantitativas (Conteo de cuadros TIMI final cTFC). (Tabla 4).

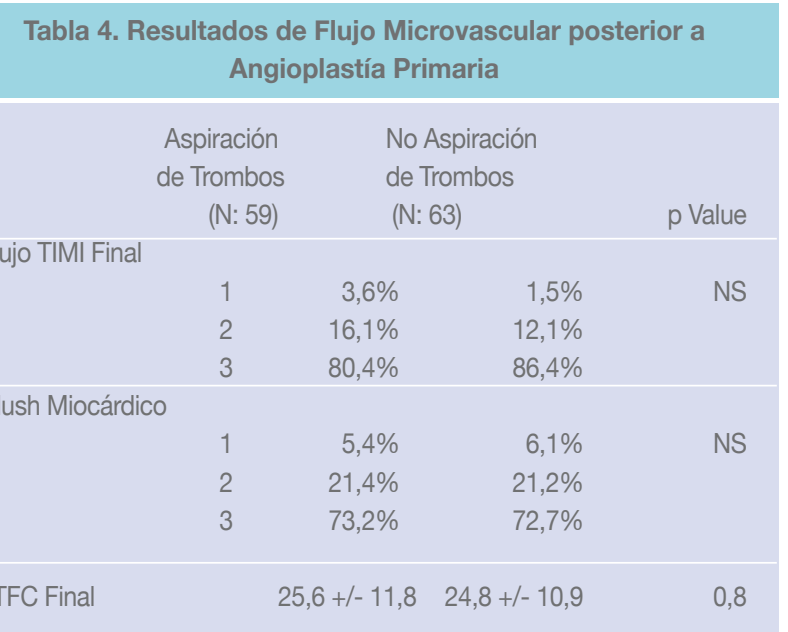

cTFC: Conteo de cuadros TIMI; NS: no significativo.

Concordante al mejor punto de predicción de curva ROC y de acuerdo a lo informado por el estudio TAPAS, se analizaron a los pacientes de acuerdo al tiempo de presentación del IAM c/SDST en $<0>3$ horas, de acuerdo al resultado de flujo microvascular, donde se objetiva que no hubo diferencias entre los grupos de aspiración de trombos vs no aspiración medido de forma cualitativa (Blush, Flujo TIMI) como cuantitativa (cTFC). Destaca la diferencia de flujo microvascular final total entre los grupos $<3$ hrs y $>3$ horas (Tabla 5).

\begin{tabular}{|c|c|c|c|}
\hline & $\begin{array}{l}\text { Aspiración } \\
\text { de Trombos } \\
\text { (N: 59) }\end{array}$ & $\begin{array}{l}\text { No Aspiración } \\
\text { de Trombos } \\
\text { (N:63) }\end{array}$ & $p$ Value \\
\hline \multicolumn{4}{|l|}{$<3$ horas } \\
\hline Flujo TIMI Final & & & NS \\
\hline 2 & $12 \%$ & $7 \%$ & \\
\hline 3 & $88 \%$ & $93 \%$ & 0.3 \\
\hline Blush Miocárdico & & & NS \\
\hline 2 & $18,2 \%$ & $14,3 \%$ & \\
\hline 3 & $81,8 \%$ & $85,7 \%$ & \\
\hline cTFC & $22,3+/-9,1$ & $22,4+/-8$ & 0.2 \\
\hline \multicolumn{4}{|l|}{$>3$ Horas } \\
\hline Flujo TIMI Final & NS & & \\
\hline 1 & $5,9 \%$ & $2,2 \%$ & \\
\hline 2 & $17,6 \%$ & $15,6 \%$ & \\
\hline 3 & $76,5 \%$ & $82,2 \%$ & \\
\hline Blush Miocárdico & & & NS \\
\hline 1 & $8,8 \%$ & $8,9 \%$ & \\
\hline 2 & $23,5 \%$ & $24,4 \%$ & \\
\hline 3 & $67,6 \%$ & $66,7 \%$ & \\
\hline $\begin{array}{l}\text { cTFC } \\
\text { NS: no Significativo }\end{array}$ & $27,7+/-13,4$ & $25,9+/-11,6$ & 0.08 \\
\hline
\end{tabular}




\section{Discusión:}

En nuestro estudio encontramos que los pacientes con IAM c/SDST tratados con angioplastía primaria y con una estrategia de aspiración rutinaria de trombos intracoronarios no hubo beneficio en términos de preservación del flujo microvascular. En el subgrupo de pacientes de presentación precoz $(<3 \mathrm{hrs}$ del IAM c/SDST), tampoco existió beneficio.

Nuestros resultados son concordantes con el estudio TAS$\mathrm{TE}^{18}$ recientemente publicado, donde no se encontró un beneficio clínico en cuanto a mortalidad, re-infarto y trombosis del stent, con un seguimiento de un año, y donde tampoco se encontró beneficio en los subgrupos estudiados (carga trombótica, vaso culpable, lesiones proximales, factores de riesgo). Esto contrasta con los estudios previos, TAPAS $^{15}$ y EXPIRA ${ }^{16-17}$, donde se había demostrado una preservación del flujo microvascular medido por Blush miocárdico y Score de ST, y en el caso del estudio EXPIRA un subgrupo demostró menor tamaño del infarto con resonancia magnética, lo que hacía pensar que la aspiración de trombos de rutina tendría impacto en outcomes clínicos. Cabe destacar que en nuestro estudio, a diferencia de los trabajos previamente mencionados, el flujo microvascular fue medido por variables cualtitativas (Flujo TIMI, Blush) y cuantitativas (cTFC), y no se usó el cTFC, lo que podría traer diferencia en los resultados obtenidos.

A pesar de no tener un beneficio de preservación del flujo microvascular en nuestros resultados, encontramos que la aspiración rutinaria de trombos podría tener un beneficio técnico, traducido en la menor necesidad de pre-dilatación de lesiones del vaso culpable y la menor necesidad de realizar técnicas complejas de bifurcación. Ello es probablemente debido a que con la aspiración se lograría una mejor visualización del vaso culpable permitiendo una angioplastía técnicamente menos compleja. A pesar de esto no hubo diferencia en los grupos en el fenómeno de no-reflow, donde uno esperaría que con menor necesidad de pre-dilatación hubiera habido una menor migración de material trombótico a distal.

Actualmente, las guías clínicas recomiendan la aspiración de rutina con un nivel de evidencia IIa, basado fundamentalmente en el estudio TAPAS ${ }^{15}$ y EXPIRA $^{16,17}$ donde se mostraba que había una mejoría a nivel microvascular posterior a la angioplastía, pero sin poder estadístico para mostrar beneficio clínico, por lo cual esta estrategia no ha podido pasar a un nivel de recomendación mayor. Desconocemos la importancia que dará el estudio $\operatorname{TASTE}^{18}$ a nivel de recomendación en las nuevas guías.

Actualmente se está desarrollando un estudio multicéntrico, prospectivo, randomizado, llamado TOTAL ${ }^{19}$, donde se reclutarán 10.700 pacientes y nos dará mayor información en cuanto al beneficio clínico de una aspiración de rutina de trombos en una angioplastía primaria.

Nuestro trabajo tiene algunas limitaciones; (1) el tamaño de nuestra muestra es pequeño comparado a los grandes trials realizados anteriormente ${ }^{15-18}$, sin embargo, con suficiente poder estadístico para poder responder la pregunta clínica de preservación de flujo microvascular con la tromboaspiración manual de rutina estratificado por horas, y aún así va en sintonía con los resultados del estudio TASTE, donde no se observó un beneficio; (2) Otra posible limitación es que el nivel de actividad antiplaquetaria no fue evaluada y podrían haber habido diferencias en ambos grupos.

En conclusión podemos decir que, en nuestra experiencia, no hay mejoría del flujo microvascular final con la tromboaspiración manual de rutina durante la AP, aún cuando la presentación del IAM c/SDST sea precoz (menor a 3 horas). La menor predilatación y el menor uso de técnicas complejas de bifurcación, podría traducir una angioplastía técnicamente más fácil al aspirar el trombo y permitir una mejor visualización del vaso culpable. 


\section{Referencias:}

1- GRINES CL, COX DA, STONE GW, GARCIA E, MATTOS LA, GIAMBARTOLOMEI A, et al. Coronary angioplasty with or without stent implantation for acute myocardial infarction. Stent Primary Angioplasty in Myocardial Infarction Study Group. N Engl J Med 1999; 341: 1949-1956.

2- Gibson CM, Cannon CP, Murphy SA, Ryan KA, Mesley, R, Marble SJ, et al. Relationship of TIMI myocardial perfusion grade to mortality after administration of thrombolytic drugs. Circulation 2000; 101: 125-130.

3- HOFFMANN R, HAAGER P, LEPPER W, FRANKE A, HANRATH P. Relation of coronary flow pattern to myocardial blush grade in patients with first acute myocardial infarction. Heart 2003; 89:1147-1151.

4- BRAUNWALD E. The treatment of acute myocardial infarction: the Past, the Present, and the Future. Eur Heart J Acute Cardiovasc Care 2012; 1: 9-12.

5- MONTALESCOT G, BARRAGAN P, WITTENBERG O, ECOLLAN P, ELHADAD, S, VILLAIN P, et al. Platelet glycoprotein IIb/IIIa inhibition with coronary stenting for acute myocardial infarction. N Engl J Med 2001; 344: 1895-1903.

6- TURER AT, HILL JA. Pathogenesis of myocardial ischemia reperfusion injury and rationale for therapy. Am J Cardiol 2010; 106: 360-368

7- STONE GW, GRINES CL, COX DA, GARCIA E, TCHENG JE, GRIFFIN JJ, et al. Comparison of angioplasty with stenting, with or without abciximab, in acute myocardial infarction. N Engl J Med 2002; 346: 957-966.

8- VAN 'T HOF AW, LIEM A, DE BOER MJ, ZIJLSTRA F. Clinical value of 12-lead electrocardiogram after successful reperfusión therapy for acute myocardial infarction. Zwolle Myocar- dial infarction Study Group. Lancet 1997; 350: 615-619.

9- WHITE CJ, RAMEE SR, COLLINS TJ, ESCOBAR AE, KARSAN A, SHAW D, et al. Coronary thrombi increase PTCA risk. Angioscopy as a clinical tool. Circulation 1996; 93: 253-258.

10- KOTANI J, NANTO S, MINTZ GS, KITAKAZE M, OHARA T, MOROZUMI T, et al. Plaque gruel of atheromatous coronary lesion may contribute to the no-reflow phenomenon in patients with acute coronary syndrome. Circulation 2002; 106: $1672-1677$

11- HENRIQUES JP, ZIJLSTRA F, OTTERVANGER JP, DE BOER MJ, VAN 'T Hof AW, HOORNTJE JC, et al. Incidence and clinical significance of distal embolization during primary angioplasty for acute myocardial infarction. Eur Heart J 2002; 23: 1112-1117.

12- VALENTE S, LAZZERI C, MATTESINI A, CHIOSTRI M, GIGLIOLI C, MEUCCI F, et al. Thrombus aspiration in elderly STEMI patients: a single center experience. Int J Cardiol 2013; 168: 3097-3099.

13- ROCHON B, CHAMI Y, SACHDEVA R, BISSETT JK, WILLIS N, URETSKY BF. Manual aspiration thrombectomy in acute ST elevation myocardial infarction: New gold standard. World J Cardiol 2011; 3: 43-47.

14- DE VITA M, BURZOTTA F, BIONDI-ZOCCAI GG, LEFEVRE T, DUDEK D, ANTONIUCCI D, et al. Individual patient-data meta-analysis comparing clinical outcome in patients with ST elevation myocardial infarction treated with percutaneous coronary intervention with or without prior thrombectomy. ATTEMPT study: a pooled Analysis of Trials on ThrombEctomy in acute Myocardial infarction based on individual PatienT data. Vasc Health Risk Manag 2009; 5: 243-247. 
15- SVILAAS T, VLAAR P, VAN DER HORST I, DIERCKS G, DE SMET BJ, VAN DEN HEUVEL FM. Thrombus Aspiration during Primary Percutaneous Coronary Intervention. (TAPAS) NEJM 2008; 358: 557-67.

16- SARDELLA G, MANCONE M, BUCCIARELLI-DUCCI C, AGATI L, SCARDALA R, CARBONE I, et al. Thrombus aspiration during primary percutaneous coronary intervention improves myocardial reperfusion and reduces infarct size: the EXPIRA (thrombectomy with export catheter in infarct-related artery during primary percutaneous coronary intervention) prospective, randomized trial. J Am Coll Cardiol 2009; 53: 309-315.

17- Sardella G, Mancone M, Canali E, Di Roma A, Benedetti G,
Stio R, et al. Impact of thrombectomy with Export Catheter in Infarct- Related Artery during Primary Percutaneous Coronary Intervention (EXPIRA Trial) on cardiac death. Am J Cardiol 2010; 106: 624-629.

18- LAGERQVIST B, FROBERT O, GORAN K, GUDNASON T, MAENG T, ALSTROM P, et al. Outcomes 1 Year after Thrombus Aspiration for Myocardial Infarction (TASTE) NEJM 2014; 371:1111-20.

19- Jolly S. A Trial of Routine Aspiration Thrombectomy With Percutaneous Coronary Intervention (PCI) Versus PCI Alone in Patients With ST-Segment Elevation Myocardial Infarction (STEMI) Undergoing Primary PCI (TOTAL). ClinicalTrials. gov Identifier: NCT01149044 